\title{
Association of GSTP1 and RRM1 Polymorphisms with the Response and Toxicity of Gemcitabine-cisplatin Combination Chemotherapy in Chinese Patients with Non-small Cell Lung Cancer
}

\author{
Zhi-Jun Yuan', Wen-Wu Zhou ${ }^{2}$, Wei Liu', Bai-Ping Wu ${ }^{3}$, Jin Zhao ${ }^{3}$, Wei Wu', \\ Yi He', Shuo Yang', Jing Su' ${ }^{1}$, Yi Luo ${ }^{1 *}$
}

\begin{abstract}
Background: Previous studies showed that genetic polymorphisms of glutathione S-transferase P1 (GSTP1) were involved in glutathione metabolism and genetic polymorphisms of ribonucleotide reductase (RRM1) were correlated with DNA synthesis. Here we explored the effects of these polymorphisms on the chemosensitivity and clinical outcome in Chinese non-small cell lung cancer (NSCLC) patients treated with gemcitabine-cisplatin regimens. Materials and Methods: DNA sequencing was used to evaluate genetic polymorphisms of GSTP1 Ile105Val and RRM1 C37A-T524C in 47 NSCLC patients treated with gemcitabine-cisplatin regimens. Clinical response was evaluated according to RECIST criteria after 2 cycles of chemotherapy and toxicity was assessed by 1979 WHO criteria (acute and subacute toxicity graduation criteria in chemotherapeutic agents). Results: There was no statistical significance between sensitive and non-sensitive groups regarding the genotype frequency distribution of GSTP1 Ile105Val polymorphism ( $p>0.05)$. But for RRM1 C37A-T524C genotype, sensitive group had higher proportion of high effective genotype than non-sensitive group $(p=0.009)$. And according to the joint detection of GSTP1 Ile105Val and RRM1 C37A-T524C polymorphisms, the proportion of type A (A/A + high effective genotype) was significantly higher in sensitive group than in non-sensitive group $(p=0.009)$. Toxicity showed no correlation with the genotypes between two groups $(p>0.05)$. Conclusions: Compared with single detection of genetic polymorphisms of GSTP1 Ile105Val or RRM1 C37A-T524C, joint detection of both may be more helpful for patients with NSCLC to receive gemcitabine-cisplatin regimens as the first-line chemotherapy. Especially, genetic polymorphism of RRM1 is more likely to be used as an important biomarker to predict the response and toxicity of gemcitabine-cisplatin combination chemotherapy in NSCLC.
\end{abstract}

Keywords: Non-small cell lung cancer - glutathione S-transferase P1 - ribonucleotide reductase subunit M1

Asian Pac J Cancer Prev, 16 (10), 4347-4351

\section{Introduction}

As we all know, variations in genes encode important proteins participating in metabolism of chemotherapeutic agents, or the target of chemotherapeutic agents. Promising results have been obtained in recent pharmacogenomics research that variations in genes may modify the efficacy of chemotherapeutic agents, thereby influencing the sensitivity and toxicity of chemotherapy for non-small cell lung cancer (NSCLC). The glutathione S-transferases (GSTs), a super-family of phase II metabolizing enzymes, play an important role in the detoxification of mutagenic and cytotoxic DNA-reactive molecules mediated by glutathione conjugation. Currently, growing evidence indicates that GSTs enzyme determines the cytotoxicity of a variety of chemotherapeutic agents (Kabler et al.,
2009; Sharma et al., 2011), especially the platinum drugs (Fuertes et al., 2003). Five subclasses of the GSTs superfamily exist, and glutathione S-transferase P1 (GSTP1) is the most abundant GSTs isoform in the lung (Singh et al., 2015; Ou et al., 2015). GSTP1, directly participating in the detoxification of platinum compounds is an important mediator of both intrinsic and acquired resistance to platinum (Parker et al., 2011; De Luca et al., 2013). Previous studies have also suggested the importance of the allelic status of GSTP1 for platinum metabolism. It is showed that at codon 105 the variants of the enzymes with valine (Val) have higher activity against cisplatin and carboplatin than enzymes with isoleucine (Ile), and hence polymorphic Ile105Val of GSTP1 is linked to the sensitivity of cancer cells to platinum (Booton et al., 2006; Lu et al., 2006; Peklak-Scott et al., 2008).

${ }^{1}$ Medical Department of Veteran Cadre, ${ }^{3}$ Molecular Biology Laboratory, Hunan Cancer Hospital, The Affiliated Cancer Hospital of Xiangya School of Medicine, Central South University, ${ }^{2}$ Department of Thoracic Surgery, Hunan Provincial People's Hospital, The First Affiliated Hospital of Hunan Normal University, Changsha, China*For correspondence: luoyi702@163.com 
Ribonucleotide reductase (RR) is a dimeric enzyme. As the major rate-limiting enzyme of DNA replication and repair, it plays an important role in deoxynucleotide production, which is a crucial step in DNA synthesis and repair. Ribonucleotide reductase subunit M1 (RRM1), the regulatory subunit of $\mathrm{RR}$, is a nucleotide-binding site. RRM1 has been identified as a potential tumor suppressor gene and controls the substrate specificity and overall activity of the enzyme. Previous data showed that RRM1 was also the primary target for gemcitabine (Qiu et al., 2014), and was involved in sensitivity to gemcitabine in NSCLC (Gray et al., 2007; Zhang et al., 2012; Li et al., 2014). Consistently, many clinical studies have demonstrated that higher levels of RRM1 are associated with gemcitabine resistance, thus proving a predictive value as well (Nakahira et al., 2007; Zhang et al., 2013; Dong et al., 2014). Recently, the research of correlation between expression levels and genetic polymorphisms of RRM1 gene and the efficacy of gemcitabine is a central issue in patients with NSCLC.

Lung cancer is one of the malignant tumors with the highest incidence and mortality in the world. Every year, NSCLC accounts for $75 \% 80 \%$ among all the patients diagnosed as lung cancer in the first time (Zhang et al., 2014). Except for a minority's cancer that could be resected, the majority of patients with NSCLC are diagnosed at an advanced stage (stage III or IV), which requires systemic-based combination chemotherapy. And one of the standard therapies for advanced NSCLC is gemcitabine-cisplatin combination chemotherapy at present (Wang et al., 2007; Wang et al., 2007). However, it is suggested by the research of modern pharmacogenetics and genomics that the genetic diversity of cells results in the heterogeneity of tumor cells, and considerable interindividual differences in therapeutic and adverse effects still exist among NSCLC patients, even with the same pathological types, clinical staging and chemotherapy. And the single neucleotide polymorphism and expression of genes are closely related to the prognosis and toxicity. The mechanism underlying the variations is still unclear. It is hypothesized that the genetic polymorphisms of genes, which code drug metabolizing enzymes or targets, may partially account for inter-individual variation in therapeutic efficacy of chemotherapy.

All in all, in view of the fact that polymorphic Ile105Val of GSTP1 is linked to the platinum metabolism, and that RRM1 expression related to the efficacy of gemcitabine is affected by RRM1 C37A-T524C polymorphism (Rodriguez et al., 2011), we aimed to assess the association of GSTP1 Ile105Val and RRM1 C37AT524C polymorphisms with the response and toxicity of gemcitabine-cisplatin combination chemotherapy in Chinese patients with NSCLC, thereby providing a molecular biological basis for clinical individualized chemotherapy in NSCLC.

\section{Materials and Methods}

\section{General data}

Forty-seven initial-treated Chinese patients (aged 29 74, with the median age of 59; 5 females and 42 males), diagnosed pathologically with advanced NSCLC, were recruited from Hunan Cancer Hospital from 2008 to 2013. All patients did not receive any other chemotherapy, but only 2 4 cycles in Medical Department of Veteran Cadre in this hospital. To avoid the confounding factor of differences in clinical staging, of 47 patients with advanced NSCLC, 27 at stage III and 20 at stage IV were included in the research. And there were 10 cases of adenocarcinoma, 33 cases of squamous carcinoma, 3 cases of adenosquamous carcinoma, and 1 case of poorly-differentiated carcinoma in total. According to Eastern Cooperative Oncology Group (ECOG) criteria, no patient's ECOG status was higher than 2, and all patients were expected to live longer than 3 months. With no severe organ damage, the status of every patient's electrocardiogram, haemogram, blood chemistries, hepatic and renal function was normal before the initiation of chemotherapy. And every patient was informed and signed an informed consent before study.

\section{Methods}

Chemotherapy regimens: All patients received gemcitabine-cisplatin combination chemotherapy for 2 4 cycles. Concrete dosage: gemcitabine $1000 \mathrm{mg} /$ $\mathrm{m} 2, \mathrm{~d} 1, \mathrm{~d} 8$; cisplatin $75 \mathrm{mg} / \mathrm{m}^{2}, \mathrm{~d} 1$ or $25 \mathrm{mg} / \mathrm{m}^{2}, \mathrm{~d} 1 \sim 3$. All chemotherapeutic drugs were injected intravenously, and the treatment cycles were repeated every 3 weeks. 128 cycles of chemotherapy were given totally, with an average of 2.7 cycles for each patient. The therapy would be terminated in case of disease progression or unacceptable toxicity.

DNA isolation and genotyping: Genomic DNA was isolated from peripheral blood leukocytes using phenol-chloroform followed by ethanol precipitation and then stored at $4^{\circ} \mathrm{C}$ before use. In this study, the genetic polymorphisms of GSTP1 Ile105Val and RRM1 C37AT524C in 47 patients with NSCLC were detected using Sanger sequencing (Chu et al., 2009). According to the results of DNA sequencing for the polymorphism of GSTP1 Ile105Val, there were homozygous for A/A wild genotype, heterozygous for $\mathrm{A} / \mathrm{G}$ genotype and homozygous for $\mathrm{G} / \mathrm{G}$ genotype; for RRM1 C37A-T524C genotype, there were high effective genotype (37AC-524CT) and non-high effective genotype (37AA-524CC, 37AA-524CT, 37AA524TT, 37AC-524CC, 37AC-524TT, 37CC-524CC, 37CC-524CT, 37CC-524TT) (Rodriguez et al., 2011). Based on the analyzed results above, the genotypes was classified into six types: A (A/A+high effective genotype), $\mathrm{B}$ (A/G+high effective genotype), C (G/G+high effective genotype), D (A/A+non-high effective genotype), E (A/ $\mathrm{G}+$ non-high effective genotype) and $\mathrm{F}$ (G/G+non-high effective genotype).

\section{Evaluation on therapeutic effects}

The patient's response to treatment was assessed every 2 cycles according to WHO criteria (response evaluation criteria in solid tumors, RECIST), which classified the response into four categories: complete remission (CR), partial remission (PR), stable disease (SD), and progressive disease (PD). Those with responses as CR and $\mathrm{PR}$, which contributed to objective response rate (ORR), 
should be verified by further examination a month later, while responses as SD and PD were considered ineffective. According to the response to chemotherapy, all patients were divided into two groups: responders (sensitive group, $\mathrm{CR}+\mathrm{PR}$ ) and non-responders (non-sensitive group, SD+PD). Toxicity was observed and assessed by 1979 WHO criteria (acute and subacute toxicity graduation criteria in chemotherapeutic agents).

\section{Statistical data analysis}

Statistical analysis was performed using SPSS 18.0 software package. The baseline characteristics between responders and non-responders were compared using $\chi^{2}$ test and Fisher probabilities. $\chi^{2}$ test and Fisher probabilities were also applied to determine the frequencies of genotypes between two groups and test the significant differences between genotypes and clinical response. Before assessing clinical association, deviations from Hardy-Weinberg equilibrium for each SNP genotype were assessed using the $\chi^{2}$ test. All statistical tests were twosided, and $p<0.05$ was considered statistically significant.

\section{Results}

Comparison on the baseline characteristics of responders and non-responders

Both genotypes of GSTP1 and RRM1 were determined in 47 patients with NSCLC. There were 2 cases of CR, 22 of PR, 19 of SD and 4 of PD. Therefore, responders and non-responders were 24 and 23 cases, respectively, and the total effective rate was $51.1 \%$. There was no statistical significance between responders and non-responders regarding the gender, age and clinical staging ( $p=0.171$, $0.308,0.292$ ), but significant difference was presented in terms of pathological types $(p=0.002)$ (Table 1$)$.

\section{GSTP1 genotype and treatment response}

The genotypic distribution frequencies of $\mathrm{G} / \mathrm{G}, \mathrm{A} / \mathrm{G}$, and A/A in GSTP1 Ile105Val polymorphism were $4.3 \%$, $31.9 \%$ and $63.8 \%$, respectively. There was no statistical significance between sensitive and non-sensitive groups regarding the gene distribution frequency of GSTP1 A $105 \mathrm{G}$ polymorphism, and no associations were detected between genotypes and response to gemcitabine-cisplatin regimens $(p=0.116)$ (Table 2$)$.

\section{RRM1 genotype and treatment response}

For RRM1 C37A-T524C, the distribution frequencies of high and non-high effective genotypes were $36.2 \%$ and $63.8 \%$, respectively. The genotype of RRM1 C37AT524C polymorphism was associated with the response to gemcitabine-cisplatin regimens, and the proportion of RRM1 C37A-T524C high effective genotype in sensitive group was higher than in non-sensitive group dramatically $(p=0.009)$ (Table 3).

Correlation between polymorphisms of GSTP1 Ile105Val and RRM1 C37A-T524C and treatment response

Because of the joint detection of GSTP1 Ile105Val and RRM1 C37A-T524C polymorphisms, 47 patients were classified into six types: A, B, C, D, E and F. And the genotypic distribution frequencies were $29.8 \%, 6.4 \%$, $0.0 \%, 34.0 \%, 25.5 \%$ and $4.3 \%$, respectively. In sensitive group, type A took the majority ( $p=0.009)$ (Table 4$)$.

\section{Correlation between polymorphisms of GSTP1 Ile105Val} and RRM1 C37A-T524C and toxicity

The major toxicities of 47 patients were gastrointestinal reactions, leucopenia, thrombocytopenia and rash, whereas there was no obvious liver and renal dysfunction.

Table 2. GSTP1 Polymorphism and Gemcitabinecisplatin Chemotherapy Response [n(\%)]

\begin{tabular}{lccccc}
\hline Groups & $\mathrm{n}$ & $\mathrm{A} / \mathrm{A}$ & $\mathrm{A} / \mathrm{G}$ & $\mathrm{G} / \mathrm{G}$ & $p$ \\
\hline Sensitive group & 24 & $16(66.67)$ & $7(29.17)$ & $1(4.17)$ & 0.116 \\
Non-sensitive group & 23 & $14(60.87)$ & $8(34.78)$ & $1(4.35)$ & \\
\hline
\end{tabular}

Table 1. Comparison on the Baseline Characteristics of Responders and Non-responders[n(\%)]

\begin{tabular}{|c|c|c|c|c|c|}
\hline Baseline Characteristics & $\mathrm{n}$ & Responders & Non-responders & $\chi^{2}$ & $p$ \\
\hline Gender & & & & 1.875 & 0.171 \\
\hline Male & 42 & $20(47.61)$ & $22(52.38)$ & & \\
\hline Female & 5 & $4(80.00)$ & $1(20.00)$ & & \\
\hline Age (years) & & & & 1.037 & 0.308 \\
\hline$<59$ & 23 & $10(43.48)$ & $13(56.52)$ & & \\
\hline$\geq 59$ & 24 & $14(58.33)$ & $10(41.67)$ & & \\
\hline Clinical staging & & & & 1.113 & 0.292 \\
\hline III & 27 & $12(44.44)$ & $15(55.56)$ & & \\
\hline IV & 20 & $12(60.00)$ & $8(40.00)$ & & \\
\hline \multicolumn{6}{|l|}{ Pathological types } \\
\hline Adenocarcinoma & 10 & $9(90.00)$ & $1(10.00)$ & 14.678 & 0.002 \\
\hline Squamous cell carcinoma & 33 & $14(42.42)$ & $19(57.58)$ & & \\
\hline Adenosquamous carcinoma & 3 & $1(33.33)$ & $2(66.67)$ & & \\
\hline Poorly-differentiated carcinoma & 1 & $0 \quad(0.00)$ & $1(100.00)$ & & \\
\hline
\end{tabular}

Table 3. RRM1 Polymorphism and Gemcitabine-cisplatin Chemotherapy Response[n(\%)]

\begin{tabular}{|c|c|c|c|c|c|c|}
\hline Groups & $\mathrm{n}$ & High effective genotype & Non-high effective genotype & $\chi^{2}$ & $p$ & $\mathrm{OR}(95 \% \mathrm{CI})$ \\
\hline Sensitive group & 24 & $13(54.17)$ & $11(45.83)$ & 6.88 & 0.009 & $5.614(1.464-21.532)$ \\
\hline Non-sensitive group & 23 & $4(17.39)$ & $19(82.61)$ & & & \\
\hline
\end{tabular}


Table 4. Combination of GSTP1 and RRM1 Polymorphisms and Gemcitabine-cisplatin Chemotherapy $\operatorname{Response[n(\% )]~}$

\begin{tabular}{lcccccc}
\hline Groups & $\mathrm{n}$ & Type A & Type D+E & $\chi^{2}$ & $p$ & OR(95\%CI) \\
\hline Sensitive group & 24 & $11(45.83)$ & $10(41.67)$ & 6.857 & 0.009 & $6.600(1.484-29.355)$ \\
Non-sensitive group & 23 & $3(13.04)$ & $18(78.26)$ & & & \\
\hline
\end{tabular}

Table 5. Combination of GSTP1 and RRM1 Polymorphisms and Toxicities of Gemcitabine-cisplatin Chemotherapy $(n=47)$

\begin{tabular}{|c|c|c|c|c|c|c|c|}
\hline Toxicities & Degrees & Type A $(n=14)$ & Type B (n=3) & Type D $(n=16)$ & Type E $(n=12)$ & Type F $(n=2)$ & $p$ \\
\hline \multirow[t]{2}{*}{ Leucopenia } & $\mathrm{I} \sim \mathrm{IV}$ & 6 & 3 & 7 & 9 & 1 & \multirow[t]{2}{*}{0.18} \\
\hline & $0^{\circ}$ & 8 & 0 & 9 & 3 & 1 & \\
\hline \multirow[t]{2}{*}{ Thrombocytopenia } & $\mathrm{I} \sim \mathrm{IV}$ & 3 & 2 & 7 & 5 & 0 & \multirow[t]{2}{*}{0.412} \\
\hline & $0^{\circ}$ & 11 & 1 & 9 & 7 & 2 & \\
\hline \multirow[t]{2}{*}{ Nausea/vomiting } & $\mathrm{I} \sim \mathrm{IV}$ & 13 & 3 & 16 & 12 & 2 & \multirow[t]{2}{*}{0.66} \\
\hline & $0^{\circ}$ & 1 & 0 & 0 & 0 & 0 & \\
\hline \multirow[t]{2}{*}{ Diarrhea } & $\mathrm{I} \sim \mathrm{IV}$ & 4 & 3 & 6 & 3 & 0 & \multirow[t]{2}{*}{0.147} \\
\hline & $0^{\circ}$ & 10 & 0 & 10 & 9 & 2 & \\
\hline \multirow{2}{*}{$\begin{array}{l}\text { Hepatic and renal } \\
\text { dysfunction }\end{array}$} & $\mathrm{I} \sim \mathrm{IV}$ & 0 & 0 & 0 & 0 & 0 & \multirow{2}{*}{-} \\
\hline & $0^{\circ}$ & 14 & 3 & 16 & 12 & 2 & \\
\hline Cardiac & $\mathrm{I} \sim \mathrm{IV}$ & 0 & 0 & 0 & 0 & 0 & \multirow[t]{2}{*}{-} \\
\hline dysfunction & $0^{\circ}$ & 14 & 3 & 16 & 12 & 2 & \\
\hline \multirow[t]{2}{*}{ Skin toxicity } & $\mathrm{I} \sim \mathrm{IV}$ & 1 & 0 & 0 & 0 & 0 & 0.66 \\
\hline & $0^{\circ}$ & 13 & 3 & 16 & 12 & 2 & \\
\hline
\end{tabular}

Toxicity showed no correlation with the genotypes of all patients $(p>0.05)$ (Table 5).

\section{Discussion}

At present, pharmacogenetics is becoming more and more popular in the study of cancer chemotherapy. Individual differences in response to cancer chemotherapy, especially drug resistance, represent a major problem. There is growing evidence implying that the polymorphisms in genes involved in metabolizing enzymes and DNA synthesis may explain the inter-individual variability of response and toxicity to chemotherapy partly (Allen et al., 2005). Therefore, assessing the correlation between genetic polymorphisms and sensitivity or resistance to chemotherapy is very important in selecting the most effective first-line treatment.

It is acknowledged that genetic polymorphisms of GSTP1 and RRM1 are involved in the metabolism of cisplatin. In this study, we attempted to explore whether these genetic polymorphisms could be used as predictors of the clinical outcome of Chinese patients with advanced NSCLC who were treated with gemcitabine-cisplatin combination chemotherapy. Certainly, some findings have been achieved in this study. Firstly, we found that GSTP1 Ile105Val mutation had no association with the clinical outcome of gemcitabine-cisplatin treatment, which was not in agreement with those from some previous studies (Sun et al., 2010; Zhou et al., 2011; Lv et al., 2012). The major reasons for this discrepancy are as follows: (1) Besides the variants of the enzyme with valine (Val) at codon 105, maybe there are some other mutations which impact on the metabolic pathways and/or efficiency of chemotherapeutic agents; (2) The sample size is relatively small and the course of treatment is short; (3) Genetic polymorphisms of GSTP1 may not play a leading role in some populations or races since the metabolism of chemotherapeutic agents is affected by many enzymes and relevant genes. Therefore, it is revealed that to study on the characteristics of the metabolism of chemotherapeutic agents, the factors including multiple relevant genes and characteristics of tumor cell DNA repair should be put into consideration for comprehensive evaluation. Secondly, the data from this study also showed that the distribution frequency of high effective genotype in 24 sensitive cases was significantly higher than in 23 non-sensitive cases, and that carriers with high effective genotype for genetic polymorphisms of RRM1 C37A-T524C in patients with NSCLC had noticeably better sensitivity. With respect to RRM1 C37A-T524C polymorphism, the result of our study was consistent with a previous study (Rodriguez et al., 2011). The potential mechanism may be that RRM1 C37A-T524C polymorphism could impact on gene expression, thereby changing the therapeutic efficacy. However, the therapeutic effect of gemcitabine-cisplatin regimens is insensitivity, even in some patients who carry with high effective genotype for genetic polymorphisms of RRM1 C37A-T524C. Hence, it further suggests that it's inappropriate to formulate individual chemotherapy program based on the characteristics of single gene. Thirdly, through the joint detection of GSTP1 Ile105Val in peripheral blood and SNP in RRM1 C37A-T524C of 47 patients with advanced NSCLC, whose genotypes could be classified into six types: A, B, C, D, E and F, it was found that the data were unevenly distributed in these types. That was mainly distributed in types A, D and $\mathrm{E}$, while no distribution in type $\mathrm{C}$. Therefore, this research focused on the analysis of the influence from gene combination of types A, D and E on chemotherapeutic efficacy. The data showed that type A was associated with chemo-sensitivity, and that toxicity had no correlation with genotypes, suggesting that high effective genotype 
for RRM1 C37A-T524C polymorphism may play a more dominant role.

Therefore, compared with single detection of genetic polymorphism in GSTP1 or RRM1, joint detection of both is more helpful for the patients with NSCLC to receive gemcitabine-cisplatin regimens as the firstline chemotherapy. Particularly, RRM1 C37A-T524C gene polymorphism is more likely to be an important biomarker for predicting the chemotherapeutic effect of patients with NSCLC to gemcitabine-cisplatin regimen, which contributes to individual therapeutic strategies of NSCLC. Further studies are necessary to predict whether our findings will be replicated in larger populations and to determine whether these differences in therapeutic efficacy of gemcitabine-cisplatin combination chemotherapy are caused by the differences in the plasma concentrations of drugs.

\section{Acknowledgements}

The research was supported by Hunan Provincial Science and Technology Department Science and Technology Plan Project Foundation (06JT3008), Hunan Provincial Science and Technology Department Vital Foundation (S2012S2032) and Scientific Research Project of Hunan Province Health Protection Special Foundation (B2012-06). We also thank the study participants.

\section{References}

Allen WL, Johnston PG (2005). Role of genomic markers in colorectal cancer treatment. J Clin Oncol, 23, 4545-52.

Booton R, Ward T, Heighway J, et al (2006). Glutathione-Stransferase P1 isoenzyme polymorphisms, platinum-based chemotherapy, and non-small cell lung cancer. $J$ Thorac Oncol, 1, 679-83.

Chu T, Bunce K, Hogge WA, et al (2009). Statistical model for whole genome sequencing and its application to minimally invasive diagnosis of fetal genetic disease. Bioinformatics, 25, 1244-50.

De Luca A, Pellizzari Tregno F, Sau A, et al (2013). Glutathione S-transferase P1-1 as a target for mesothelioma treatment. Cancer Sci, 104, 223-30.

Dong X, Hao Y, Wei Y, et al (2014). Response to first-line chemotherapy in patients with non-small cell lung cancer according to RRM1 expression. PLoS One, 9, 92320.

Fuertes MA, Castilla J, Alonso C, et al (2003). Cisplatin biochemical mechanism of action: from cytotoxicity to induction of cell death through interconnections between apoptotic and necrotic pathways. Curr Med Chem, 10, 257-66.

Gray J, Simon G, Bepler G (2007). Molecular predictors of chemotherapy response in non-small-cell lung cancer. Expert Rev Anticancer Ther, 7, 545-9.

Kabler SL, Seidel A, Jacob J, et al (2009). Differential protection by human glutathione S-transferase P1 against cytotoxicity of benzo[a]pyrene, dibenzo[a,1]pyrene, or their dihydrodiol metabolites, in bi-transgenic cell lines that co-express rat versus human cytochrome P4501A1. Chem Biol Interact, 179, 240-6.

Li Y, Wang LR, Chen J, et al (2014). First-line gemcitabine plus cisplatin in non-small cell lung cancer patients. Dis Markers, 2014, 960458.

Lu C, Spitz MR, Zhao H, et al (2006). Association between glutathione S-transferase pi polymorphisms and survival in patients with advanced non-small cell lung carcinoma. Cancer, 106, 441-7.

Lv HY, Li QC, Wei HJ, et al (2012). Relationship between GSTP1 and XPG genetic polymorphisms and survival of platinum-based chemotherapy in advanced non-small cell lung cancer patients. China Oncology, 22, 609-17.

Nakahira S, Nakamori S, Tsujie M, et al (2007). Involvement of ribonucleotide reductase M1 subunit overexpression in gemcitabine resistance of human pancreatic cancer. Int $J$ Cancer, 120, 1355-63.

Ou C, Zhu B, Zhao HL, et al (2015). Association of glutathione S-transferase P1 gene polymorphism with the risk of smallcell carcinoma of lungcancer. J Recept Signal Transduct Res, 35, 165-9.

Parker LJ, Italiano LC, Morton CJ, et al (2011). Studies of glutathione transferase P1-1 bound to a platinum(IV)-based anticancer compound reveal the molecular basis of its activation. Chemistry, 17, 7806-16.

Peklak-Scott C, Smitherman PK, Townsend AJ, et al (2008). Role of glutathione S-transferase P1-1 in the cellular detoxification of cisplatin. Mol Cancer Ther, 7, 3247-55.

Qiu ZQ, Zhao K (2014). Expression of ERCC1, RRM1 and LRP in non-small cell lung cancers and their influence on chemotherapeutic efficacy of gemcitabine concomitant with nedaplatin. Asian Pac J Cancer Prev, 15, 7303-7.

Rodriguez J, Boni V, Hernández A, et al (2011). Association of RRM1 -37A $>$ C polymorphism with clinical outcome in colorectal cancer patients treated with gemcitabine-based chemotherapy. Eur J Cancer, 47, 839-47.

Sharma R, Ellis B, Sharma A (2011). Role of alpha class glutathione transferases (GSTs) in chemoprevention: GSTA1 and A4 overexpressing human leukemia (HL60) cells resist sulforaphane and curcumin induced toxicity. Phytother Res, 25, 563-8.

Singh S (2015). Cytoprotective and regulatory functions of glutathione S-transferases in cancer cell proliferation and cell death. Cancer Chemother Pharmacol, 75, 1-15.

Sun N, Sun X, Chen B, et al (2010). MRP2 and GSTP1 polymorphisms and chemotherapy response in advanced non-small cell lung cancer. Cancer Chemother Pharmacol, 65, 437-46.

Wang LR, Huang MZ, Zhang GB, et al (2007). Phase II study of gemcitabine and carboplatin in patients with advanced non-small-cell lung cancer. Cancer Chemother Pharmacol, 60, 601-7.

Wang L, Wu X, Huang M, et al (2007). The efficacy and relationship between peak concentration and toxicity profile of fixed-dose-rate gemcitabine plus carboplatin in patients with advanced non-small-cell lung cancer. Cancer Chemother Pharmacol, 60, 211-8.

Zhang GB, Chen J, Wang LR, et al (2012). RRM1 and ERCC1 expression in peripheral blood versus tumor tissue in gemcitabine/carboplatin-treated advanced non-small cell lung cancer. Cancer Chemother Pharmacol, 69, 1277-87.

Zhang X, Jin FS, Zhang LG, et al (2013). Predictive and prognostic roles of ribonucleotide reductase M1 in patients with pancreatic cancer treated with gemcitabine: a metaanalysis. Asian Pac J Cancer Prev, 14, 4261-5.

Zhang Y, Yu L, Xia N (2014). Effect of Brucea Javanica Oil Emulsion combined with GP regimen on the immune function of patients with advanced non-small cell lung cancer. J Int Transl Med, 2, 262-5.

Zhou F, Yu Z, Jiang T, et al (2011). Genetic polymorphisms of GSTP1 and XRCC1: prediction of clinical outcome of platinum-based chemotherapy in advanced non-small cell lung cancer (NSCLC) patients. Swiss Med Wkl, 141, 13275. 